\title{
Warm and hot circumstellar gas in V1647 Ori during the 2008-2009 outburst
}

\author{
A. Carmona ${ }^{1, *}$, M. Audard ${ }^{1}$, M. van den Ancker $^{2}$, G. van der Plas ${ }^{2,3}$, \\ M. Goto ${ }^{4}$ and D. Fedele ${ }^{4}$ \\ ${ }^{1}$ ISDC \& Geneva Observatory, ch. d'Ecogia 16, 1290 Versoix, Switzerland \\ email: andres.carmona@unige.ch \\ ${ }^{2}$ European Southern Observatory, Karl Schwarzschild Str 2, D-85748 Garching, Germany \\ ${ }^{3}$ University of Amsterdam, Kruislaan 403, NL-1098 SJ Amsterdam, The Netherlands \\ ${ }^{4}$ Max-Planck Institute for Astronomy, Königstuhl 17, D-69117 Heidelberg, Germany \\ ${ }^{*}$ M.A and A.C acknowledge support from a Swiss National Science Foundation grant \\ (PP002-110504). Based on observations at the ESO-VLT (program ID: DDT 281C-5056).
}

\begin{abstract}
The pre-main sequence star V1647 Ori started a new outburst in August 2008. From October 2008 to February 2009 we monitored V1647 Ori, obtaining quasi-simultaneous VLT-CRIRES near-IR spectroscopy, VLT-VISIR mid-IR spectroscopy and VLT-FORS2 optical spectroscopy. We studied the evolution of $\mathrm{H}_{2}$ and $\mathrm{CO}$ emission from hot and warm gas and $\mathrm{H} \alpha$ and forbidden line-emission during the initial outburst phase of V1647 Ori. $\mathrm{H} \alpha$ is observed in emission displaying P-Cygni profiles with blue-shifted absorption up to $-700 \mathrm{~km} / \mathrm{s}$, suggesting the presence of a high velocity wind (Fig. 1a). [OI] emission at $6300 \AA$ is observed displaying a blue-shifted emission shoulder, indicating the presence of material moving away from the star (Fig. 1b). We detect $\mathrm{H}_{2}$ 1-0 S(1) and $\mathrm{CO}$ (P4 to P14 and P30-P38) ro-vibrational lines centered at the velocity of the star at all epochs (Fig. 1c \& d). This strongly suggests that the $\mathrm{H}_{2}$ and $\mathrm{CO}$ emission originates from a disk and not from a warm outflow. The $\mathrm{H}_{2}$ 1-0 S(0) and 2-1 $\mathrm{S}(1)$ ro-vibrational lines at 2.22 and $2.24 \mu \mathrm{m}$ and the pure-rotational $\mathrm{H}_{2} 0-0 \mathrm{~S}(1)$ and $0-0 \mathrm{~S}(2)$ lines at 17 and $12 \mu \mathrm{m}$ were not detected in our spectra. Changes in the $\mathrm{H} \alpha$ and [OI] profiles and the $\mathrm{H}_{2}$ and $\mathrm{CO}$ emission observed do not correlate. We modeled the $\mathrm{H}_{2}$ and $\mathrm{CO}$ line profiles assuming emission from a flat disk in keplerian rotation with line intensity decreasing with radius $\left(I \sim I_{0}\left(R / R_{\mathrm{min}}\right)^{-\alpha}\right)$. We found that the disk of V1647 Ori is observed nearly face-on and that the line emission is produced within a fraction of an AU of the star (Fig. 1d).
\end{abstract}

Keywords. stars: pre-main-sequence, circumstellar matter, individual (V1647 Ori)

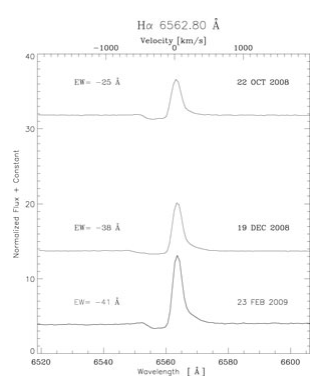

a.

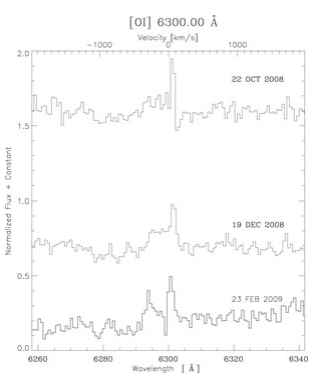

b.

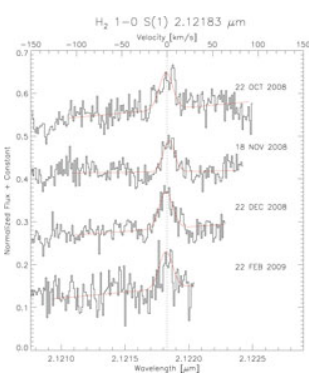

c.

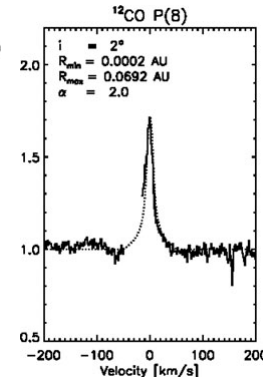

d.

Figure 1. Panels $a, b \& c: \mathrm{H} \alpha,[\mathrm{OI}]$ and $\mathrm{H}_{2}$ 1-0 $\mathrm{S}(1)$ spectra observed. Panel $d$ : Keplerian flat disk model of the CO $\mathrm{P}(8)$ line (Carmona et al. in preparation). 